\title{
The Role of Mesolimbic Reward Neurocircuitry in Prevention and Rescue of the Activity-Based Anorexia (ABA) Phenotype in Rats
}

\author{
Claire J Foldi', Laura K Milton' and Brian J Oldfield*,I \\ 'Department of Physiology, Monash University, Clayton, VIC, Australia
}

\begin{abstract}
Patients suffering from anorexia nervosa (AN) become anhedonic; unable or unwilling to derive normal pleasures and avoid rewarding outcomes, most profoundly in food intake. The activity-based anorexia (ABA) model recapitulates many of the characteristics of the human condition, including anhedonia, and allows investigation of the underlying neurobiology of AN. The potential for increased neuronal activity in reward/hedonic circuits to prevent and rescue weight loss is investigated in this model. The mesolimbic pathway extending from the ventral tegmental area (VTA) to the nucleus accumbens (NAc) was activated using a dual viral strategy, involving retrograde transport of Cre (CAV-2-Cre) to the VTA and coincident injection of DREADD receptors (AAV-hSyn-DIO-hM3D(Gq)-mCherry). Systemic clozapine-n-oxide (CNO; $0.3 \mathrm{mg} / \mathrm{kg}$ ) successfully recruited a large proportion of the VTA-NAc dopaminergic projections, with activity evidenced by colocalization with elevated levels of Fos protein. The effects of reward circuit activation on energy balance and predicted survival was investigated in female Sprague-Dawley rats, where free access to running wheels was paired with time-limited (90 min) access to food, a paradigm (ABA) which will cause anorexia and death if unchecked. Excitation of the reward pathway substantially increased food intake and food anticipatory activity (FAA) to prevent ABA-associated weight loss, while overall locomotor activity was unchanged. Similar activation of reward circuitry, delayed until establishment of the ABA phenotype, rescued rats from their precipitous weight loss. Although these data are consistent with shifts primarily in food intake, the contribution of mechanisms including energy expenditure to survival remains to be determined. These results will inform the neurobiological underpinnings of $\mathrm{AN}$, and provide insight into the mechanisms of reward circuitry relevant to feeding and weight loss.

Neuropsychopharmacology (2017) 42, 2292-2300; doi: 10.1038/npp.2017.63; published online 19 April 2017
\end{abstract}

\section{INTRODUCTION}

Anorexia nervosa (AN) is a disorder with complex etiology and the highest mortality rate of any psychiatric disease (Harris and Barraclough, 1998). AN is characterized by a dramatic reduction in body weight caused by excessive caloric restriction, which is accompanied by physiological, biochemical and behavioral disturbances including the diminished capacity to experience pleasure or reward (Klein and Walsh, 2004). These patients have been described as anhedonic or ascetic (Davis and Woodside, 2002). A lack of understanding of the pathophysiology of AN has hindered the development of effective treatments, with no clear evidence to support the efficacy of existing pharmacotherapeutic interventions beyond improving the depressive symptoms (SSRIs) or delusional dysmorphic features (olanzapine and haloperidol) that often complicate the course of the disorder (Miniati et al, 2016).

* Correspondence: Professor BJ Oldfield, Department of Physiology, Monash University, 26 Innovation Walk, Clayton, VIC 3800, Australia, Tel: +6I 3990 52507. Fax: +6I 399020707.

E-mail: brian.oldfield@monash.edu

Received 24 January 2017; revised 23 February 2017; accepted I March 2017; accepted article preview online 21 March 2017
Although genetic, sociocultural and psychosocial factors undoubtedly contribute to the pathogenesis of $\mathrm{AN}$, it is now clear that there are underlying neurobiological drivers (Gorwood et al, 2016; Kaye et al, 2013; van Kuyck et al, 2009). Indeed, mounting evidence from neuroimaging studies supports a role for altered central reward processing in the development and maintenance of AN (Frank et al, 2012; Frank et al, 2016b; Kaye et al, 2009; O'Hara et al, 2015). For example, patients with AN have reduced neural activity in the striatum in response to sucrose (Wagner et al, 2008) and increased dopamine (DA) receptor binding in the anterior ventral striatum (indicating decreased endogenous DA concentration (Frank et al, 2005)) compared to healthy controls. In addition, a disrupted dopaminergic system may play a role in susceptibility to $\mathrm{AN}$, with multiple DA receptor polymorphisms associated with the condition (Bergen et al, 2005). The link between anorexia and anhedonia is highlighted by studies showing that patients with AN dislike highly palatable foods (Fernstrom et al, 1994), rate food as negative when hungry (Santel et al, 2006) and report dysphoric mood following food consumption (Kaye, 2008). 
Anhedonia has its biological underpinnings in the mesolimbic DA system (Davis and Woodside, 2002) and it is therefore plausible that disrupted DA signaling in rewardbased neural circuits gives rise to anhedonia in AN (DerAvakian and Markou, 2012). The microstructure of neural circuits and transmitters coding for reward can be simply subdivided into (1) the activation of $\mu$ opioid, endocannabinoid or GABA receptors in the nucleus accumbens (NAc), which enhance the affective value or hedonic 'liking' characteristics of reward; and (2) dopaminergic projections originating in the ventral tegmental area (VTA) and extending to the NAc which encode for motivated behaviors (Berridge and Kringelbach, 2008; Der-Avakian and Markou, 2012).

Activity-based anorexia (ABA) is a robust and widely recognized rodent model, which mimics many of the important characteristics of human AN (Dixon et al, 2003), including hyperactivity and reduced food consumption and, of particular relevance to the present study, anhedonia (Brown et al, 2008; Verty et al, 2011). In the ABA paradigm, unrestricted access to running wheels (RW) and time-limited access to food leads to a profound reduction in body weight that, without intervention, results in death by starvation (Epling et al, 1983; Routtenberg and Kuznesof, 1967). Several lines of evidence implicate disrupted DA signaling and anhedonia as central to the ABA phenotype. First, DA antagonism with cis-flupenthixol reduces body weight loss associated with ABA by increasing food intake and reducing hyperactivity (Verhagen et al, 2009a). Furthermore, $\Delta^{9}$-tetrahydrocannabinol (THC) increases DA levels in the striatum (Malone and Taylor, 1999), and in ABA rats THC administration increases highly palatable food (high-fat diet; HFD) consumption leading to attenuation of body weight loss (Verty et al, 2011). Moreover, access to highly palatable food alone (sweet HFD), that is known to activate neurochemistry associated with reward, can both prevent and rescue ABA-associated body weight loss (Brown et al, 2008). This suggests that increasing the hedonic value of food is pertinent to prevention of and recovery from the $\mathrm{ABA}$ phenotype in rats.

The combination of designer receptor exclusively activated by designer drugs (DREADD) technologies with Cremediated homologous recombination enables specific targeting and manipulation of neural pathways (Gore et al, 2013). Activation of the VTA-NAc pathway by DREADD-Cre technology has been shown to markedly increase neuronal firing of predominantly dopaminergic neurons and is associated with behaviorally relevant consequences (Boekhoudt et al, 2016; Boender et al, 2014). Given the evidence of disrupted reward in $\mathrm{AN}$ and $\mathrm{ABA}$, the present study was performed to determine (1) whether activation of the VTA-NAc pathway using DREADD technology can prevent the development of the ABA phenotype and (2) whether rats exposed to the ABA paradigm can be rescued from further weight loss by activation of this pathway.

\section{MATERIALS AND METHODS}

\section{Animals and Housing}

Female Sprague-Dawley rats (Animal Resources Centre, Western Australia) weighing 130-160 g were used in these studies (approved by the Monash Animal Resource Platform ethics committee; MARP/2015/046). Animals were allowed to lose a total of $20-25 \%$ of baseline body weight before being removed from the experiment and euthanized with $300 \mathrm{mg} /$ $\mathrm{kg}$ sodium pentobarbitone (Lethabarb; Virbac, Australia). Rats were housed individually in transparent activity wheel and living chambers (Lafayette Instruments, model no. $80859)$ in a temperature $\left(22.5-25^{\circ} \mathrm{C}\right)$ and humidity $(60 \pm 10 \%)$ controlled room, under a $12 \mathrm{~h}$ light/dark cycle (lights off at 15:00 hours). A singly housed male rat was present in the experimental room to synchronize the estrous cycles of the female rats.

\section{Viral Activation Strategy}

A dual viral strategy was employed, involving microinjection into two brain regions in the same rat. CAV-2-Cre ${ }^{\text {EGFP }}$ (IGMM; Montpellier, France) was injected into the NAc with subsequent retrograde transport to the VTA, amongst other well defined sources of afferent input to the NAc. AAV-hSynDIO-hM ${ }_{3} \mathrm{D}(\mathrm{Gq})$-mCherry (abbreviated $+\mathrm{M}_{3}$-mCherry; UNC Vector Core, USA) was injected into the VTA and contains the floxed inverted sequence of $\mathrm{hM}_{3} \mathrm{D}(\mathrm{Gq})$-mCherry, which reorients in the presence of Cre to allow DREADD receptor expression and ligand binding. This ensures that $+\mathrm{M}_{3^{-}}$ mCherry is not expressed in all AAV-infected cells, but exclusively in those that are also infected with CAV-2Cre ${ }^{\text {EGFP }}$, thus defining the VTA to NAc neuronal pathway. $+\mathrm{M}_{3}$-mCherry is activated by clozapine-n-oxide (CNO; Tocris Bioscience), delivered via daily intraperitoneal injection $(0.3 \mathrm{mg} / \mathrm{kg})$, causing infected cells to depolarize.

\section{Surgical and Viral Injection Procedures}

All rats were injected bilaterally with $250 \mathrm{nl}$ of CAV-2-Cre ${ }^{\text {EGFP }}\left(5.0 \times 10^{8}\right.$ genomic copies/ $\mu$ l) into NAc (from bregma: AP: $+1.6 \mathrm{~mm}, \mathrm{ML}: \pm 0.8 \mathrm{~mm}$, and $\mathrm{DV}$ : $-6.6 \mathrm{~mm}$ ) using a stereotaxic apparatus (Kopf Instruments, CA, USA). Rats then received bilateral injections of $250 \mathrm{nl}$ of $+\mathrm{M}_{3}$-mCherry $\left(4.5 \times 10^{12}\right.$ genomic copies/ $\left.\mu \mathrm{l}\right)$ into VTA (from bregma; AP: $\quad-5.3 \mathrm{~mm}, \mathrm{ML}: \pm 0.6 \mathrm{~mm}$, DV: $-7.7 \mathrm{~mm}$ ). Viruses were infused over $5 \mathrm{~min}$ through glass micropipettes with a tip diameters $\sim 40 \mu \mathrm{m}$. Both viruses were injected under the same anesthetic $(2.5 \%$ isoflurane in oxygen), with $\sim 30 \mathrm{~min}$ between CAV-2-Cre and $+\mathrm{M}_{3}$-mCherry infusions and $5 \mathrm{~min}$ after each injection to allow virus dispersal. Preliminary studies revealed no noticeable shift in efficacy of recombination when CAV-2Cre was allowed 7-10 days for transport before +M3mCherry injections - as such all subsequent paired injections were made as described above, coincidentally. Meloxicam $(0.2 \mathrm{mg} / \mathrm{kg}$, s.c.; Boehringer Ingelheim, Germany) and Enrofloxacin (25 mg/ml in drinking water; Bayer, Germany) were administered to all rats for $48 \mathrm{~h}$ post-surgery. Viral injections were conducted at least 10 days before the experimental period, to allow for virus transport and recombination.

\section{Running Wheel Activity Measurement}

Running wheel activity (RWA) in cage mounted wheels was recorded with Activity Wheel Software (Lafayette 
Instruments, IN, USA), at 10 min intervals for the duration of the experiment. Baseline RWA was measured for $48 \mathrm{~h}$ before the commencement of ABA (or food restriction) and these values were considered when allocating animals to treatment groups so that there was no significant difference in RWA between the groups before the experiment.

\section{Evaluation of the Microstructure of ABA}

Over a 15 day period, food (standard laboratory rodent chow; Barastoc Feeds, AU) was provided ad libitum for 5 days (Ad lib), restricted to $90 \mathrm{~min} /$ day for 5 days (food restriction; FR), and ad libitum for a further 5 days (Recovery). Comparing rats with RWs $(n=6)$ and locked RWs (LRW; $n=6$ ) over these feeding schedules allowed examination of changes in body weight associated with (a) access to a RW alone (b) food restriction alone and (c) the combination of RW access and food restriction.

\section{Effect of Ventral Reward Circuit Activation on Development of ABA}

To determine the effects of ventral reward circuit activation on the development of ABA, rats $(N=24)$ were acclimatized for a period of 7 days with ad libitum food and unlimited access to RWs, and were allocated to treatment groups matched for body weight and baseline RWA. On day 1 of ABA, all food was removed from the cages and rats were weighed and injected with either $\mathrm{CNO}$ or saline within the period $30 \mathrm{~min}$ before food presentation. All rats had ad libitum access to water, voluntary access to RWs, and intermittent access $(90 \mathrm{~min} / \mathrm{day}$, at the onset of the dark phase) to food for the experimental period. The ABA protocol was maintained until rats reached $80 \%$ of baseline body weight or for a maximum of 10 days. On reaching either of these endpoints, animals were anesthetized with Lethabarb and perfused transcardially with $200 \mathrm{ml}$ saline followed by $200 \mathrm{ml} \mathrm{4 \%}$ paraformaldehyde in phosphate buffer (PFA-PB) and brains were excised for processing. For Fos expression analysis, animals ( $n=4 \mathrm{CNO}, n=4$ saline) were injected i.p. 90 min before perfusion.

\section{Effect of Ventral Reward Circuit Activation on Recovery from $\mathrm{ABA}$}

To determine the effects of ventral reward circuit activation on recovery from $\mathrm{ABA}$, delivery of $\mathrm{CNO}$ or saline was delayed until after rats $(N=20)$ had lost at least $14.5 \%$ of their baseline body weight. This threshold of body weight loss was determined from the prevention experiment as the mean minimum body weight for rats that survived the paradigm, regardless of treatment group. As rats approached this body weight loss criterion, they were allocated by alternation to be either maintained on saline (control) or switched to $\mathrm{CNO}$ (rescue) administration. In this way, an even distribution of animals were designated to each group before post-mortem exclusions. As above, when rats reached body weight loss criteria for removal from the experiment, they were transcardially perfused and brains excised for processing.

\section{Immunohistochemistry and Imaging}

Brains were post-fixed in 4\% PFA-PB solution overnight at $4{ }^{\circ} \mathrm{C}$, followed by submersion in $30 \%$ sucrose in $\mathrm{PB}$ solution for 3-4 days. Brains were sectioned at $35 \mu \mathrm{m}$ using a cryostat (CM1860, Leica Biosystems); sections were collected in multiwell cell culture plates in a 1:4 series and stored in cryoprotectant before standard immunohistochemical processing using the following primary antibodies for $24 \mathrm{~h}$ at room temperature: chicken anti-EGPF (1:2000, Abcam), mouse anti-DsRed (1:500, Santa Cruz), and rabbit anti-cfos (1:2000, Santa Cruz). Subsequently, sections were incubated for $90 \mathrm{~min}$ in secondary antibodies conjugated with Alexa Fluor dyes 488, 594 and 647 (1:500, Abcam). Confirmation of successful injection placement was determined post mortem by virtue of the localization of the fluorescent reporters associated with the viruses, which revealed the extent of Cre and DREADD labeling in the VTA. CNO-treated rats with brains containing fewer than 10 mCherry-labeled neurons per section were excluded from all subsequent analyses.

Imaging was conducted using a Leica SP5 confocal microscope $(\times 20$ objective magnification, $1024 \times 1024$ resolution) running LAS AF software (Leica MicroSystems. Manheim, Germany). Tiled, $z$-stacked images $(4 \times 2$ tiles, $z=9$ ) were captured of the ventral portion of the brain, containing VTA, from $4 \times 35 \mu \mathrm{m}$ sections from each animal. mCherry-labeled neurons in the VTA were counted using Image J software (National Institutes of Health, USA) and confirmation of activation was assessed by colocalization with Fos expression. To determine whether DREADDexpressing VTA cells were dopaminergic, triple immunostaining was performed on a subset of CNO-treated brains $(n=4)$ using anti-EGFP, anti-DsRed and an antibody against tyrosine hydroxylase (rabbit anti-TH, 1:1000; Abcam) as a marker for identification of dopaminergic neurons.

\section{Statistical Analyses}

Independent samples $t$-test was used to compare the proportion of double-labeled VTA cells $\left(+\mathrm{M}_{3}-\mathrm{mCherry} / \mathrm{Fos}\right)$ in CNO vs saline-treated rats. Survival was evaluated with a log-rank (Mantel-Cox) $\chi^{2}$ test. To enable longitudinal analysis of the group data, in which cases are progressively removed (a necessary consequence of successful ABA), the data were restructured in SPSS using the VARSTOCASES command generated from the Restructure Data Wizard and then analyzed with a custom SPSS syntax (Supplementary Figure 1). Mixed model ANOVAs followed by post hoc multiple comparisons with a Bonferroni correction were used to analyze body weight, food intake and RWA. Independent samples $t$-tests were used to analyze mean daily food intake and mean daily RWA. Linear regression was used to determine the relationships between food intake, body weight loss, and RWA. Significance for all tests was set at $p<0.05$.

\section{RESULTS}

\section{ABA Only Develops with the Combination of Food Restriction and Running Wheel Access}

To define the individual impact of the two components of the $\mathrm{ABA}$ procedure, restricted food availability and RW 
access were varied independently. Following FR, there was an $\sim 10 \%$ reduction in body weight to a plateau that was maintained during the FR period (middle, Figure 1a). There was an immediate increase in body weight following the return to free food availability, with a trajectory that matched that of body weight gain in the initial Ad Lib feeding period (right, Figure 1a).

When only RW access was varied between the two groups (Ad Lib), all rats displayed the normal increase in body weight that would be expected over this period (left, Figure 1a). The impact of access to RWs on body weight only became evident during FR, in which RW rats showed a significant reduction in body weight compared to LRW rats $\left(\mathrm{F}_{1,10}=12.32, p=0.006\right)$ (middle, Figure 1a). When returned to ad libitum food (right, Figure 1a) there was a recovery of the trajectory of body weight gain for both groups, however, the RW group remained significantly lighter during the Recovery period.

Running wheel access only influenced food intake when food was available ad libitum. RW rats ate less than LRW rats on the first 2 days of the Ad Lib phase (left panel; Figure 1b) and more than LRW rats during the Recovery period (right panel; Figure 1b, all $p<0.01$ ). RW rats displayed a remarkably similar pattern of activity throughout the phases of food availability, with the expected hyperactivity during FR shown by an increase and peak in RWA within the 5 -day food restriction period for 5/6 rats (middle panel; Figure 1c).

\section{CNO Administration Activates DREADD-Infected VTA Neurons}

Visualization of immunolabelled-mCherry revealed that DREADD labeling was restricted to the VTA, with no mCherry expression in substantia nigra or surrounding areas (Figure 2a). Colocalization of $+\mathrm{M}_{3}$-mCherry with Fos protein (a marker for neuronal activity) demonstrated that $\mathrm{CNO}$ $(0.3 \mathrm{mg} / \mathrm{kg})$ activates a majority $(67.63 \pm 5.84 \%)$ of $+\mathrm{M}_{3^{-}}$ mCherry-labeled neurons, over and above the residual Fos activation $(3.76 \pm 2.58 \%)$ in saline-treated controls $\left(t_{6}=20.01, p<0.0001\right.$; Figures $\left.2 \mathrm{~b}-\mathrm{d}\right)$. Immunostaining for TH revealed that the majority of targeted VTA neurons $(73.96 \pm 5.19 \%)$ were dopaminergic (data not shown).

\section{Ventral Reward Circuit Activation Prevents Development of the ABA Phenotype}

To determine the effects of ventral reward circuit activation on body weight maintenance during $\mathrm{ABA}$, rats that had undergone viral infusions were administered either $\mathrm{CNO}$ (activated) or saline (control) daily for the entire ABA paradigm. Animals were allocated to treatment groups such that there was no significant differences in mean body weight between groups at the start of the experiment $\left(t_{22}=0.11\right.$, $p=0.92)$. When $\mathrm{CNO}$ was administered daily from the start of the ABA protocol, a significant increase in survival was observed $\left(80 \%\right.$ vs $14.3 \% ; \chi^{2}(1)=9.95, p=0.002$; Figure $\left.3 a\right)$, which reflected a profound attenuation of body weight loss (Figure 3b).

Average daily RWA showed a phasic response with an increase and peak in RWA during the dark phase and a decrease in RWA during the light phase, which was largely
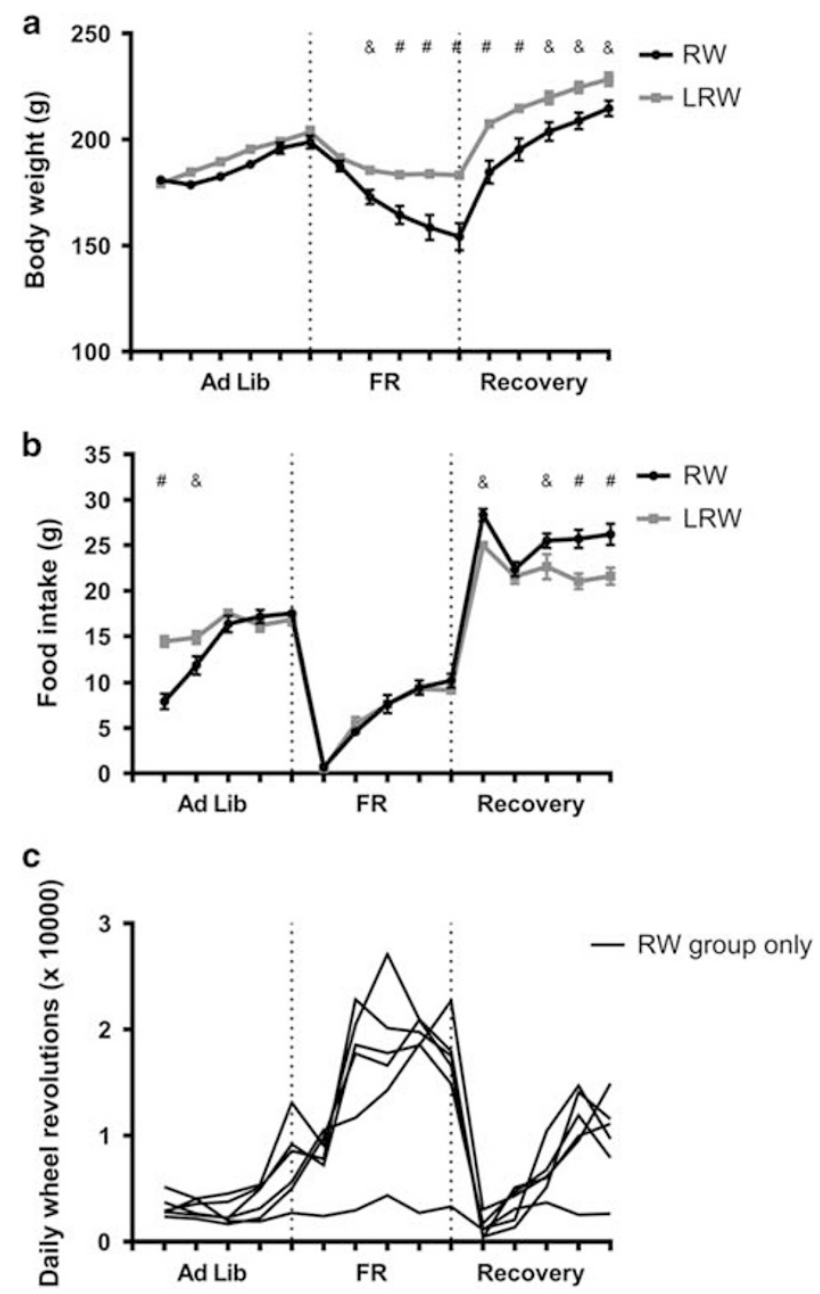

Figure I Effects of food availability on (a) body weight trajectory, (b) daily food intake and (c) running wheel activity (RWA) for rats with locked (LRW, $n=6$ ) or unlocked (RW, $n=6$ ) running wheels. Results are expressed as mean \pm SEM $\left({ }^{\&} p<0.01,{ }^{\#} p<0.001\right)$.

unchanged by ventral reward circuit activation $\left(\mathrm{F}_{1,21}=\right.$ $0.001, p=0.975)$. However, during the hour preceding food presentation (14:00-15:00 hours), a significant increase in RWA was observed after reward circuit activation, consistent with enhanced food anticipatory activity (FAA; $\mathrm{F}_{1,297.6}=8.44, p=0.004$; Figure $3 \mathrm{c}$ ).

Activation of the ventral reward circuit elevated food consumption trajectory, resulting in significantly more food consumed daily, on average, throughout the ABA paradigm $\left(t_{22}=3.43, p=0.002\right.$; Figure $\left.3 d\right)$.

\section{Delayed Ventral Reward Circuit Activation Promotes Survival of ABA}

To determine whether the ABA phenotype could be rescued by ventral reward circuit activation, rats that had undergone viral infusions were administered only saline until they reached $85.5 \%$ of their baseline body weight. Rats were then administered either CNO (activated) or saline (control). As above, mean body weight was not significantly different between groups at the start of this experiment $\left(t_{19}=0.71\right.$, $p=0.49)$. Delayed CNO administration increased survival 


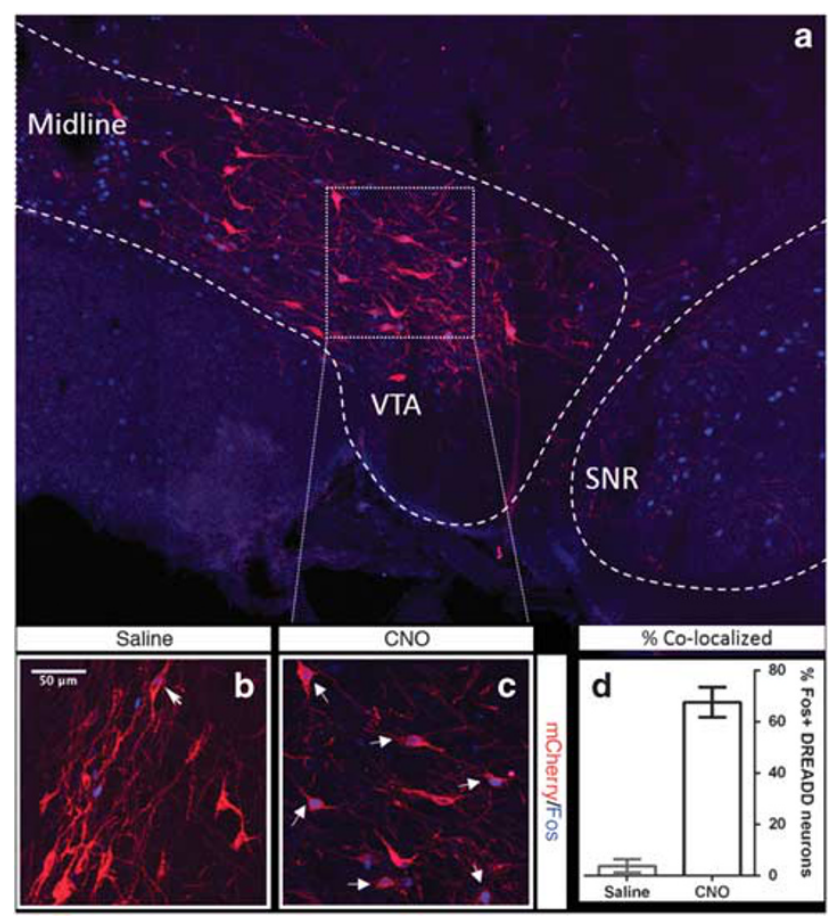

Figure 2 Designer receptor exclusively activated by designer drugs (DREADD) expression is restricted to the ventral tegmental area (VTA) (a) and colocalization of Fos and mCherry labeling within VTA is evident in the majority of clozapine-n-oxide (CNO)-treated, DREADD-labeled cells, over and above the residual activation seen in saline-treated animals ( $b$ and $c$ ), validating our in vivo DREADD activation strategy $(p<0.0000 \mathrm{l}$; d; mean \pm SEM). White dashed lines indicate the contours of the VTA and substantia nigra (SNR).

$\left(\chi^{2}(1)=3.97, p=0.046\right.$; Figure $\left.4 a\right)$ by attenuating further body weight loss (Figure $4 \mathrm{~b}$ ) without any significant effects on RWA $\left(F_{1,20.15}=0.061, p=0.81\right.$; Figure $\left.4 c\right)$. Mean daily food intake over the course of the experiment was not significantly increased after ventral reward circuit activation $\left(t_{19}=1.56, p=0.13\right.$; Figure $\left.4 d\right)$.

\section{DISCUSSION}

The most important finding of this study is that the dramatic body weight free fall that accompanies ABA can be ameliorated by increasing neuronal activity in the midbrain reward circuit, utilizing pathway-specific DREADD technologies.

We have also reevaluated the microstructure of the ABA protocol, namely the interrelationship of time-limited food availability and RWA. The fact that these individually cause no substantial reduction in body weight and only impact anorexic behavior in combination is an essential tenet of the paradigm, however, only recently has systematic testing of this assumption become routine (Chen et al, 2016; Scharner et al, 2016). Here, we confirmed that when time-limited food access was combined with RW access, initial body weight loss was accompanied by rapid-onset hyperactivity, leading to the body weight 'free fall' characteristic of ABA, consistent with a considerable net energy expenditure.

The present finding that chemogenetic activation of the ventral reward pathway, extending from VTA to NAc, attenuates body weight loss to both prevent and rescue the
ABA phenotype is consistent with other observations suggesting an involvement of reward pathways in this model (Avena and Bocarsly, 2012; Brown et al, 2008; Verty et al, 2011). It is also in line with the proposal that human $A N$ is founded in an anhedonia (Davis and Woodside, 2002; O'Hara et al, 2015), involving reward pathways (Frank et al, 2005; Kaye et al, 2009; Park et al, 2014). Previous studies have indicated that rodent $\mathrm{ABA}$ can be ameliorated by increasing the hedonic or rewarding value of food, by substituting laboratory chow with a HFD alone (Brown et al, 2008) or combined with THC administration (Verty et al, 2011), both of which have effects mediated by DA signaling (Malone and Taylor, 1999). While provision of a HFD undoubtedly increased survival in the ABA model, the effects were not intrinsic to the animal but rather a modification to food type. This does not translate to an applicable treatment option for the human condition, because patients with anorexia find high-fat energy-dense food aversive (Kaye, 2008). The results of the present study offer an alternative by suggesting that increasing neuronal activity in reward circuitry can increase food intake without changing the reward context of the food itself. It is likely, however, that both the studies using THC and/or HFD and the present experiments using reward circuit activation influence outcomes in ABA via the same underlying mechanisms.

There is a widespread assumption that mesolimbic pathways, including the VTA-NAc pathway, are primarily dopaminergic (Bjorklund and Dunnett, 2007). We report that the majority (74\%) of DREADD-labeled VTA cells colocalized with $\mathrm{TH}$, in line with previous reports using similar methodologies (Boekhoudt et al, 2016; Boender et al, 2014). The DREADD-labeled cells that do not express TH likely represent a separate population of neurons with candidates including those that are primarily GABAergic or glutamatergic (Morales and Margolis, 2017). Regardless, given the very high representation of TH in the DREADDlabeled population and the likelihood that immunocytochemical detection represents an underestimate of the total population, it is reasonable to assume that the recruited VTA-NAc pathways in the current experiments were predominantly dopaminergic. We therefore suggest that, by increasing the firing rate of VTA-NAc neurons, reward circuit activation increases DA availability in the NAc to either enhance the rewarding value of food or increase motivated approach behavior to the salient food stimulus.

The neurochemistry of the circuitry underlying locomotor activity and feeding is complex, involving multiple monoamine and opioid systems (Avena and Bocarsly, 2012), however, these behaviors are widely considered to be, in part, mediated by midbrain DA signaling (Zhang et al, 2003). For example, mice lacking $\mathrm{TH}$ in dopaminergic neurons become hypophagic (Zhou and Palmiter, 1995) and rats treated with 6-hydroxydopamine (6-OHDA) to selectively deplete DA neurons are both hypoactive and hypophagic (Zigmond and Stricker, 1972). Furthermore, DA receptor antagonism in rats leads to decreases in both food intake and locomotor activity (Pitts and Horvitz, 2000) as well as decreased FAA (Liu et al, 2012). Conversely, DA receptor agonism in rats with ad libitum food access can also decrease meal size and duration (Cooper et al, 2006) and decrease intake of both chow and HFD foods (McQuade et al, 2003). With respect to ABA, systemic treatment with DA antagonists has been 

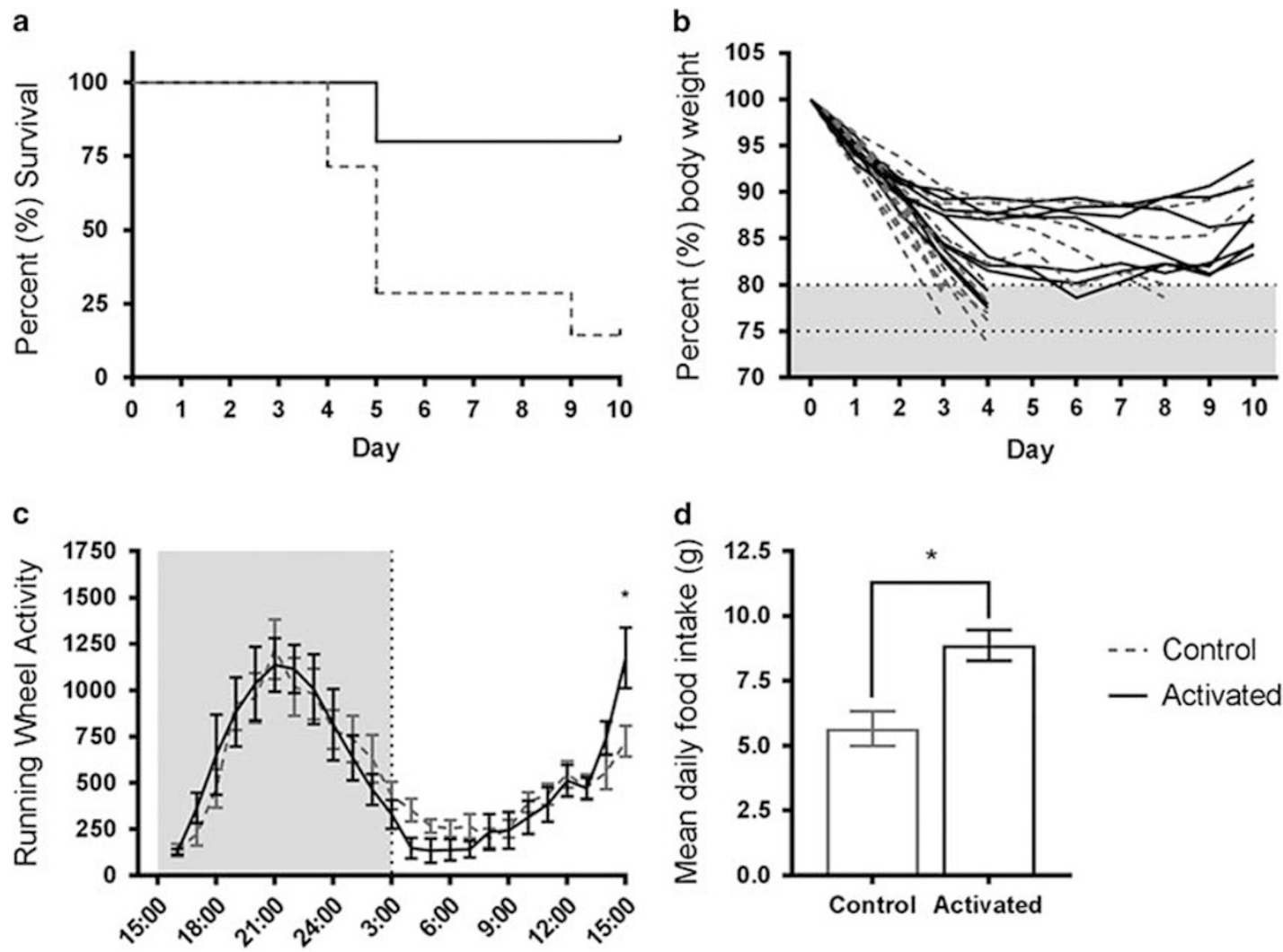

Figure 3 Survival (a), body weight loss trajectory (b), average daily running wheel activity (RWA) (c) and mean daily food intake (d) in rats treated with clozapine-n-oxide (CNO; activated, $n=10$ ) or saline (control, $n=14$ ) to prevent activity-based anorexia (ABA). Results expressed as mean $\pm \mathrm{SEM}$, $* 2<0.0$ I.
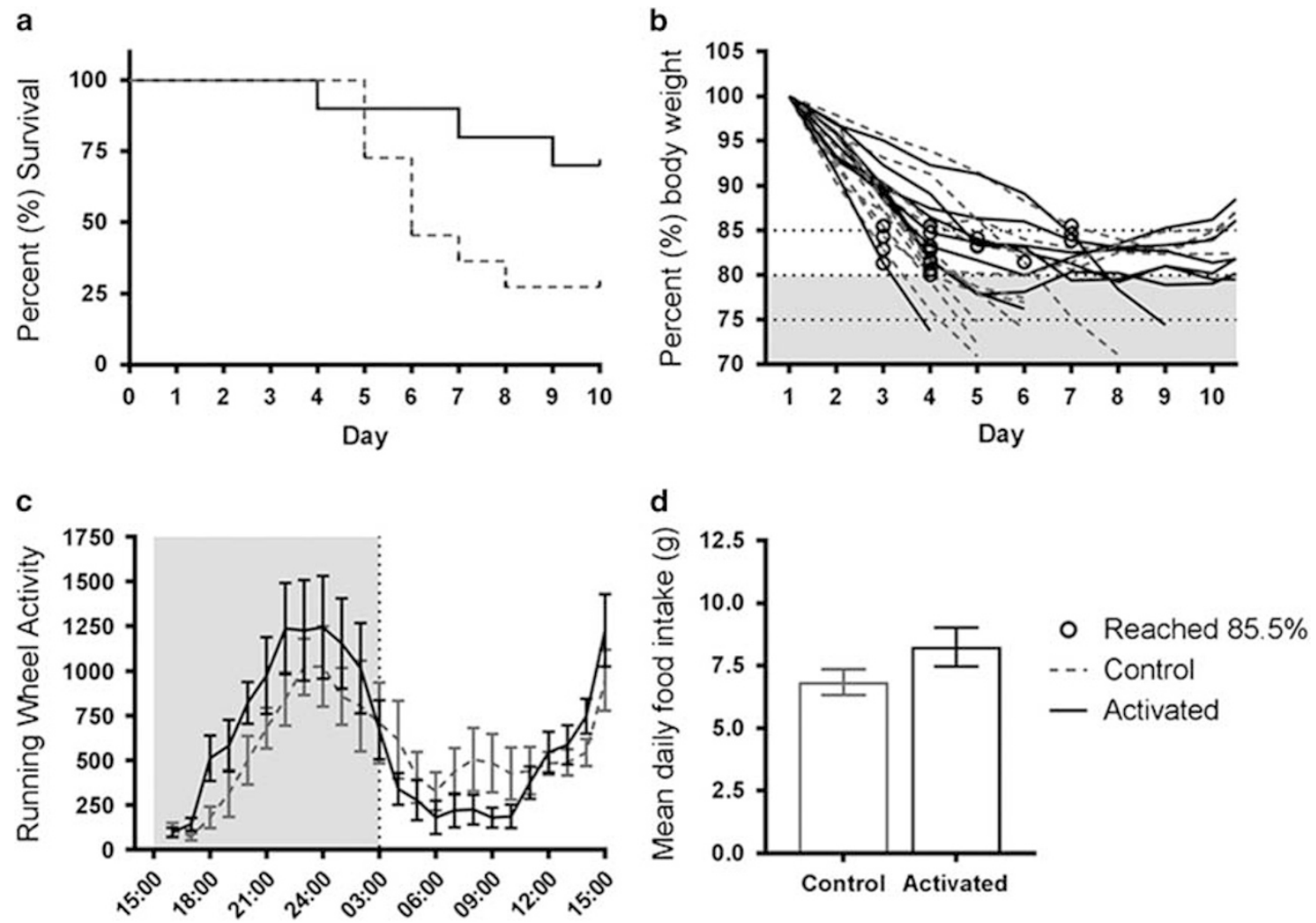

Figure 4 Survival (a), body weight loss trajectory (b), average daily running wheel activity (RWA) (c) and mean daily food intake (d) in rats treated with clozapine-n-oxide (CNO; activated, $n=9$ ) or saline (control, $n=I I)$ to rescue activity-based anorexia (ABA). CNO administration was delayed until rats reached at least $85.5 \%$ baseline body weight (indicated with circles in $b$ ). Results expressed as mean \pm SEM. 
shown to promote body weight maintenance and survival of ABA by increasing food intake or suppressing hyperactivity (Klenotich et al, 2015; Verhagen et al, 2009a). This suggests that ABA can be ameliorated by decreasing DA signaling and is at odds with the present data. These contradictions highlight the complexity of global vs pathway-specific DA effects on food intake in the ad libitum and restricted feeding environments. Evidence suggests that food intake during ABA is associated with increased DA signaling, specifically within the midbrain reward pathway, based on the observation that extracellular DA release in NAc increases during feeding in ABA with no similar increase seen for periods of hyperactivity (Verhagen et al, 2009b). Consistent with this, the results of the present study suggest that VTA-NAc activation increases DA availability in the NAc to promote food intake in the context of altered reward, with no effect on RWA. Putting these data in the context of the human condition it should be appreciated that while disrupted DA signaling contributes to the disorder (Frank et al, 2005), manipulation of dopaminergic circuits, despite their proposed utility (Frank, 2014), have been ineffective in the treatment of AN to date (Miniati et al, 2016).

The question arises as to whether perturbation of midbrain reward pathways will impact more on food intake or physical activity, both of which, based on the human comparison, could be considered 'rewarding'. Activation of the midbrain reward pathway increased daily food intake, which prevented ABA-associated body weight loss. There was no overall effect of VTA-NAc pathway activation on RWA, indicating a specific effect on food reward over 'reward' in a more general sense. Correlation analyses also support this, with daily food intake accounting for $63 \%$ of the variation in body weight loss, whereas RWA accounted for only $23 \%$ of this variation (Supplementary Figure 2). FAA in response to timed food restriction is seen commonly in ABA models (Wu et al, 2014) and has been proposed as an adaptive response to food scarcity and the anticipation of finding food (Epling and Pierce, 1988). The present study revealed FAA was elevated after midbrain reward circuit activation, with no overall effects on RWA, further strengthening the hypothesis that specific activation of the VTA-NAc pathway in the context of ABA is associated with increasing the reward of or motivation for food rather than any reward intrinsic to running. That is, if rats associate food with reward circuit activation, then they are more likely to anticipate food presentation with FAA. This is in agreement with previous reports of increased FAA in response to palatable over chow food presentation (Barbano and Cador, 2006) and elevated extracellular DA levels in the NAc during feeding in ABA but not FAA (Verhagen et al, 2009b).

While the data presented here focus on food intake as the driving force in the amelioration of weight loss, the extent of the contribution from energy expenditure remains unresolved. This is particularly important when considering that delayed activation of the VTA-NAc pathway to rescue an established ABA phenotype did not significantly alter running activity $(p=0.81)$ or food intake $(p=0.13)$. One possibility is that reward circuit activation during ABA alters some other aspect of energy balance, such as brown adipose tissue (BAT) thermogenesis, which contributed to the amelioration of body weight loss. There is evidence to suggest that the reward system is directly involved in BAT function, because markers of thermogenic activity (UCP1 protein) in BAT are reduced by THC administration and presentation of HFD during ABA (Verty et al, 2011). Therefore, it is conceivable that increasing the activity of VTA-NAc in the present study might reduce the thermogenic activity of BAT, which would decrease energy expenditure and promote survival.

These data highlight the importance of the activation of mesolimbic circuitry in the amelioration of ABA in a rodent model and open the door to further studies examining the role of the interrelationship with related hypothalamic and frontal cortical pathways in this process. In this respect, it should be recognized that there exists a hypothesis for the underpinnings of the human condition involving an interplay between hedonic drivers of food intake (and other rewards) and powerful inhibitory influences from prefrontal cortex (Ehrlich et al, 2015; Frank et al, 2016a). Individuals recovered from $\mathrm{AN}$, compared to controls, demonstrate exaggerated neural activity in the dorsolateral prefrontal cortex in anticipation of reward (Ehrlich et al, 2015), reduced activity in striatum in response to sweet taste (Wagner et al, 2008) and altered activity in the insula (Frank et al, 2016a), an area of the brain involved in interoceptive awareness. Taken together, this indicates that altered eating in AN may be a consequence of aberrant reward processing in the context of heightened cognitive control processes, with interoceptive deficits leading to poor awareness of homeostatic needs (Park et al, 2014). As with all studies of rodent models of human conditions, the hope will be to uncover a targetable component of these brain circuitries that is essential to the development of ABA and possibly AN. Any such focus would represent a profound impact on the amelioration of a condition that currently has no effective treatment.

\section{FUNDING AND DISCLOSURE}

The authors declare no conflict of interest.

\section{ACKNOWLEDGMENTS}

We acknowledge the use of facilities at Monash Micro Imaging, Monash University, Victoria, Australia.

\section{REFERENCES}

Avena NM, Bocarsly ME (2012). Dysregulation of brain reward systems in eating disorders: neurochemical information from animal models of binge eating, bulimia nervosa, and anorexia nervosa. Neuropharmacology 63: 87-96.

Barbano MF, Cador M (2006). Differential regulation of the consummatory, motivational and anticipatory aspects of feeding behavior by dopaminergic and opioidergic drugs. Neuropsychopharmacology 31: 1371-1381.

Bergen AW, Yeager M, Welch RA, Haque K, Ganjei JK, van den Bree MB et al (2005). Association of multiple DRD2 polymorphisms with anorexia nervosa. Neuropsychopharmacology 30: 1703-1710.

Berridge KC, Kringelbach ML (2008). Affective neuroscience of pleasure: reward in humans and animals. Psychopharmacology (Berl) 199: 457-480. 
Bjorklund A, Dunnett SB (2007). Dopamine neuron systems in the brain: an update. Trends Neurosci 30: 194-202.

Boekhoudt L, Omrani A, Luijendijk MC, Wolterink-Donselaar IG, Wijbrans EC, van der Plasse G et al (2016). Chemogenetic activation of dopamine neurons in the ventral tegmental area, but not substantia nigra, induces hyperactivity in rats. Eur Neuropsychopharmacol 26: 1784-1793.

Boender AJ, de Jong JW, Boekhoudt L, Luijendijk MC, van der Plasse G, Adan RA (2014). Combined use of the canine adenovirus-2 and DREADD-technology to activate specific neural pathways in vivo. PLoS ONE 9: e95392.

Brown AJ, Avena NM, Hoebel BG (2008). A high-fat diet prevents and reverses the development of activity-based anorexia in rats. Int J Eat Disord 41: 383-389.

Chen YW, Actor-Engel H, Sherpa AD, Klingensmith L, Chowdhury TG, Aoki C (2016). NR2A- and NR2B-NMDA receptors and drebrin within postsynaptic spines of the hippocampus correlate with hunger-evoked exercise. Brain Struct Funct (e-pub ahead of print).

Cooper SJ, Al-Naser HA, Clifton PG (2006). The anorectic effect of the selective dopamine D1-receptor agonist A-77636 determined by meal pattern analysis in free-feeding rats. Eur J Pharmacol 532: 253-257.

Davis C, Woodside DB (2002). Sensitivity to the rewarding effects of food and exercise in the eating disorders. Compr Psychiatry 43: 189-194.

Der-Avakian A, Markou A (2012). The neurobiology of anhedonia and other reward-related deficits. Trends Neurosci 35: 68-77.

Dixon DP, Ackert AM, Eckel LA (2003). Development of, and recovery from, activity-based anorexia in female rats. Physiol Behav 80: 273-279.

Ehrlich S, Geisler D, Ritschel F, King JA, Seidel M, Boehm I et al (2015). Elevated cognitive control over reward processing in recovered female patients with anorexia nervosa. J Psychiatry Neurosci 40: 140249.

Epling FW, Pierce DW (1988). Actvity-based anorexia: a biobehavioral perspective. Int J Eat Disord 7: 475-485.

Epling FW, Pierce DW, Stefan L (1983). A theory of activity-based anorexia. Int J Eat Disord 3: 27-46.

Fernstrom MH, Weltzin TE, Neuberger S, Srinivasagam N, Kaye WH (1994). Twenty-four-hour food intake in patients with anorexia nervosa and in healthy control subjects. Biol Psychiatry 36: $696-702$.

Frank GK (2014). Could dopamine agonists aid in drug development for anorexia nervosa? Front Nutr 1: 19.

Frank GK, Bailer UF, Henry SE, Drevets W, Meltzer CC, Price JC et al (2005). Increased dopamine D2/D3 receptor binding after recovery from anorexia nervosa measured by positron emission tomography and [11c]raclopride. Biol Psychiatry 58: 908-912.

Frank GK, Collier S, Shott ME, O'Reilly RC (2016a). Prediction error and somatosensory insula activation in women recovered from anorexia nervosa. J Psychiatry Neurosci 41: 304-311.

Frank GK, Reynolds JR, Shott ME, Jappe L, Yang TT, Tregellas JR et al (2012). Anorexia nervosa and obesity are associated with opposite brain reward response. Neuropsychopharmacology 37: 2031-2046.

Frank GK, Shott ME, Riederer J, Pryor TL (2016b). Altered structural and effective connectivity in anorexia and bulimia nervosa in circuits that regulate energy and reward homeostasis. Transl Psychiatry 6: e932.

Gore BB, Soden ME, Zweifel LS (2013). Manipulating gene expression in projection-specific neuronal populations using combinatorial viral approaches. In: Jacqueline NC (ed). Current Protocols In Neuroscience 4: 4.35.1-4.35.20.

Gorwood P, Blanchet-Collet C, Chartrel N, Duclos J, Dechelotte P, Hanachi $\mathrm{M}$ et al (2016). New insights in anorexia nervosa. Front Neurosci 10: 256.
Harris EC, Barraclough B (1998). Excess mortality of mental disorder. Br J Psychiatry 173: 11-53.

Kaye W (2008). Neurobiology of anorexia and bulimia nervosa. Physiol Behav 94: 121-135.

Kaye WH, Fudge JL, Paulus M (2009). New insights into symptoms and neurocircuit function of anorexia nervosa. Nat Rev Neurosci 10: $573-584$.

Kaye WH, Wierenga CE, Bailer UF, Simmons AN, Bischoff-Grethe A (2013). Nothing tastes as good as skinny feels: the neurobiology of anorexia nervosa. Trends Neurosci 36: 110-120.

Klein DA, Walsh BT (2004). Eating disorders: clinical features and pathophysiology. Physiol Behav 81: 359-374.

Klenotich SJ, Ho EV, McMurray MS, Server CH, Dulawa SC (2015). Dopamine D2/3 receptor antagonism reduces activity-based anorexia. Transl Psychiatry 5: e613.

Liu YY, Liu TY, Qu WM, Hong ZY, Urade Y, Huang ZL (2012). Dopamine is involved in food-anticipatory activity in mice. J Biol Rhythms 27: 398-409.

Malone DT, Taylor DA (1999). Modulation by fluoxetine of striatal dopamine release following Delta9-tetrahydrocannabinol: a microdialysis study in conscious rats. $\mathrm{Br} J$ Pharmacol 128: 21-26.

McQuade JA, Benoit SC, Woods SC, Seeley RJ (2003). 7-OH-DPAT selectively reduces intake of both chow and high fat diets in different food intake regimens. Pharmacol Biochem Behav 76: 517-523.

Miniati M, Mauri M, Ciberti A, Mariani MG, Marazziti D, Dell'Osso L (2016). Psychopharmacological options for adult patients with anorexia nervosa. CNS Spectr 21: 134-142.

Morales M, Margolis EB (2017). Ventral tegmental area: cellular heterogeneity, connectivity and behaviour. Nat Rev Neurosci 18: $73-85$.

O'Hara CB, Campbell IC, Schmidt U (2015). A reward-centred model of anorexia nervosa: a focussed narrative review of the neurological and psychophysiological literature. Neurosci Biobehav Rev 52: 131-152.

Park RJ, Godier LR, Cowdrey FA (2014). Hungry for reward: How can neuroscience inform the development of treatment for Anorexia Nervosa? Behav Res Ther 62: 47-59.

Pitts SM, Horvitz JC (2000). Similar effects of $D(1) / D(2)$ receptor blockade on feeding and locomotor behavior. Pharmacol Biochem Behav 65: 433-438.

Routtenberg A, Kuznesof AW (1967). Self-starvation of rats living in activity wheels on a restricted feeding schedule. J Comp Physiol Psychol 64: 414-421.

Santel S, Baving L, Krauel K, Munte TF, Rotte M (2006). Hunger and satiety in anorexia nervosa: fMRI during cognitive processing of food pictures. Brain Res 1114: 138-148.

Scharner S, Prinz P, Goebel-Stengel M, Kobelt P, Hofmann T, Rose $M$ et al (2016). Activity-based anorexia reduces body weight without inducing a separate food intake microstructure or activity phenotype in female rats-mediation via an activation of distinct brain nuclei. Front Neurosci 10: 475.

van Kuyck K, Gerard N, Van Laere K, Casteels C, Pieters G, Gabriels L et al (2009). Towards a neurocircuitry in anorexia nervosa: evidence from functional neuroimaging studies. J Psychiatr Res 43: 1133-1145.

Verhagen LA, Luijendijk MC, Hillebrand JJ, Adan RA (2009a). Dopamine antagonism inhibits anorectic behavior in an animal model for anorexia nervosa. Eur Neuropsychopharmacol 19: 153-160.

Verhagen LA, Luijendijk MC, Korte-Bouws GA, Korte SM, Adan RA (2009b). Dopamine and serotonin release in the nucleus accumbens during starvation-induced hyperactivity. Eur Neuropsychopharmacol 19: 309-316.

Verty AN, Evetts MJ, Crouch GJ, McGregor IS, Stefanidis A, Oldfield BJ (2011). The cannabinoid receptor agonist THC 
attenuates weight loss in a rodent model of activity-based anorexia. Neuropsychopharmacology 36: 1349-1358.

Wagner A, Aizenstein H, Mazurkewicz L, Fudge J, Frank GK, Putnam K et al (2008). Altered insula response to taste stimuli in individuals recovered from restricting-type anorexia nervosa. Neuropsychopharmacology 33: 513-523.

Wu H, van Kuyck K, Tambuyzer T, Luyten L, Aerts JM, Nuttin B (2014). Rethinking food anticipatory activity in the activity-based anorexia rat model. Sci Rep 4: 3929.
Zhang M, Balmadrid C, Kelley AE (2003). Nucleus accumbens opioid, GABaergic, and dopaminergic modulation of palatable food motivation: contrasting effects revealed by a progressive ratio study in the rat. Behav Neurosci 117: 202-211.

Zhou QY, Palmiter RD (1995). Dopamine-deficient mice are severely hypoactive, adipsic, and aphagic. Cell 83: 1197-1209.

Zigmond MJ, Stricker EM (1972). Deficits in feeding behavior after intraventricular injection of 6-hydroxydopamine in rats. Science 177: $1211-1214$.

Supplementary Information accompanies the paper on the Neuropsychopharmacology website (http://www.nature.com/npp) 